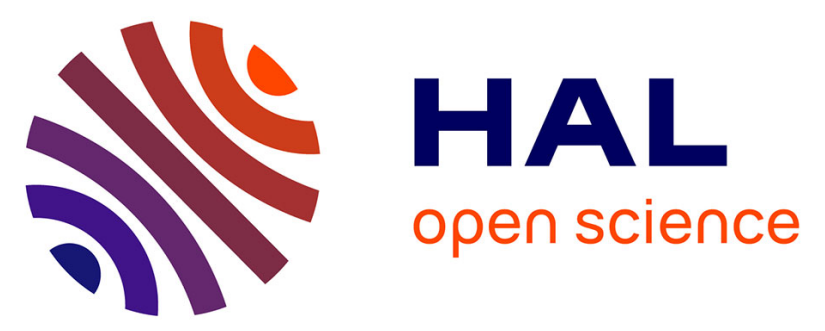

\title{
Sorafenib plus octreotide is an effective and safe treatment in advanced hepatocellular carcinoma: multicenter phase II So.LAR. study
}

Salvatore Del Prete, Liliana Montella, Michele Caraglia, Luigi Maiorino, Gregorio Cennamo, Vincenzo Montesarchio, Guido Piai, Antonio Febbraro, Luciano Tarantino, Elena Capasso, et al.

\section{To cite this version:}

Salvatore Del Prete, Liliana Montella, Michele Caraglia, Luigi Maiorino, Gregorio Cennamo, et al.. Sorafenib plus octreotide is an effective and safe treatment in advanced hepatocellular carcinoma: multicenter phase II So.LAR. study. Cancer Chemotherapy and Pharmacology, 2009, 66 (5), pp.837844. 10.1007/s00280-009-1226-z . hal-00568278

\section{HAL Id: hal-00568278 https://hal.science/hal-00568278}

Submitted on 23 Feb 2011

HAL is a multi-disciplinary open access archive for the deposit and dissemination of scientific research documents, whether they are published or not. The documents may come from teaching and research institutions in France or abroad, or from public or private research centers.
L'archive ouverte pluridisciplinaire HAL, est destinée au dépôt et à la diffusion de documents scientifiques de niveau recherche, publiés ou non, émanant des établissements d'enseignement et de recherche français ou étrangers, des laboratoires publics ou privés. 
SORAFENIB PLUS OCTREOTIDE IS AN EFFECTIVE AND SAFE TREATMENT IN ADVANCED HEPATOCELLULAR CARCINOMA: MULTICENTER PHASE II SO.LAR. STUDY

Salvatore Del Prete ${ }^{1}$, Liliana Montella ${ }^{1}$, Michele Caraglia ${ }^{3}$, Luigi Maiorino ${ }^{2}$, Gregorio Cennamo ${ }^{1}$, Vincenzo Montesarchio ${ }^{4}$, Guido Piai $^{5}$, Antonio Febbraro ${ }^{6}$, Luciano Tarantino $^{7}$, Elena Capasso ${ }^{8}$, Giovannella Palmieri ${ }^{10}$, Rosario Guarrasi ${ }^{1}$, Maddalena Bianco $^{11}$, Rosanna Mamone ${ }^{12}$, Clementina Savastano ${ }^{13}$, Agata Pisano ${ }^{14}$, Bruno Vincenzi ${ }^{15}$, Antonietta Sabia ${ }^{16}$, Alberto D’Agostino ${ }^{17}$, Vincenzo Faiola ${ }^{1}$, Raffaele Addeo ${ }^{1}$.

${ }^{1}$ Medical Oncology Unit, "San Giovanni di Dio" Hospital, Frattaminore, Naples; ${ }^{2}$ Department of Biochemistry and Biophysics, Second University of Naples, Naples; ${ }^{3}$ Oncology Unit "San Gennaro" Hospital, Naples; " Medical Oncology "Cotugno" Hospital, Naples; ${ }^{5}$ AO San Sebastiano, Caserta; ${ }^{6}$ Medical Oncology, Fatebenefratelli, Benevento; ${ }^{7}$ Hepatology and Interventional Ultrasound Unit, "San Giovanni di Dio" Hospital, Frattaminore, Naples; ${ }^{8}$ Senology Unit, Arzano, Naples; ${ }^{9}$ UO di Ecografia Interventistica IX Divisione, “Cotugno” Hospital, Naples; ${ }^{10}$ Department of Molecular and Clinical Endocrinology \& Oncology, University "Federico II", Naples; ${ }^{11 ، S a n}$ Leonardo" Hospital, Castellammare di Stabia, ASL NA5, Naples; ${ }^{12}$ Radiology and Diagnostic Imaging Unit “San Giovanni di Dio" Hospital, Frattaminore, Naples; ${ }^{13}$ Medical Oncology “Ruggi d'Aragona” Hospital, Salerno; ${ }^{14}$ Medical Oncology “S.Maria delle Grazie" Hospital, Pozzuoli, Naples; ${ }^{15}$ Medical Oncology Campus Biomedico, Roma; ${ }^{16}$ Medical Oncology Unit, "S.Maria della Pietà”, Nola, Italy; ${ }^{17}$ Surgery Division Unit, “Loreto Mare”, Naples, Italy 
Corresponding Author: Salvatore Del Prete, Medical Oncology Unit, "San Giovanni di Dio” Hospital, Via Giovanni XXII, 80020 Frattaminore, Naples, E-mail saldelprete@yahoo.it; tel +390818891334 fax +390818891353

Running title: Sorafenib and octreotide in HCC

Keywords: Sorafenib, long acting Octreotide; hepatocellular carcinoma; combination therapy 


\section{ABSTRACT}

Purpose. Advanced hepatocellular carcinoma (HCC) not eligible for local therapies has limited chances of cure. Sorafenib is a multi-kinase inhibitor with proven activity in advanced HCC. Octreotide is used in this setting with conflicting results. Treatment with sorafenib and long-acting octreotide was tested in advanced HCC to evaluate safety and activity. Methods. Fifty patients with advanced HCC, Child-Pugh A or B, received sorafenib at a dosage of $800 \mathrm{mg} /$ daily for 28 days with a following week of rest and long acting octreotide at a dose of $40 \mathrm{mg}$, administered every 28 days. Results. All patients were assessable for safety and efficacy. Sixteen patients out of 50 (34\%) were naïve from other therapies, while all the others were previously treated with local and/or systemic treatments. We achieved 5 partial responses (10\%), 33 stable diseases $(66 \%)$ and 12 progressions of disease (24\%). Median time to progression was 7.0 months (95\% CI, 3.0 to 10.9 months) and median overall survival was 12 months (95\% CI, 6.3 to 17.4 months). Treatment was well tolerated. Diarrhoea (6\%) and hypertension (4\%) were the most frequent grade 3 toxicities. Conclusions. Our data suggest that the combination between sorafenib and long acting octreotide is active and well tolerated in patients with advanced HCC and could represent another efficacious chance for the management of this population. 


\section{INTRODUCTION}

HCC is the fifth most common cancer in the world, and the third most common cause of cancer-related death (1).

In order to reduce the morbidity and mortality of $\mathrm{HCC}$, early diagnosis and the development of novel systemic therapies for advanced disease, including drugs, gene and immune therapies as well as primary HCC prevention are of paramount importance. Sorafenib (BAY 43-9006) is an oral multikinase inhibitor that is effective as a singleagent therapy in renal cell carcinoma and was recently approved for treatment of advanced HCC. Sorafenib is a novel oral bis-aryl urea compound originally developed as an inhibitor to RAF kinase for its anti-proliferative property. It also inhibits receptor tyrosine kinases of multiple pro-angiogenic factors such as VEGFR-2/3, Flt-3/ and PDGFR-beta (2). Sorafenib demonstrated a good safety profile and encouraging antitumor effects when used with other agents in patients with advanced solid tumors (3).

In SHARP trial 602 patients with advanced HCC were randomly assigned to sorafenib at a dose of $400 \mathrm{mg}$ twice daily or placebo (4). Median overall survival was 10.7 months in the sorafenib group and 7.9 months in the placebo group (hazard ratio in the sorafenib group, $0.69 ; 95 \%$ confidence interval, 0.55 to $0.87 ; \mathrm{P}<0.001)$. In Asia-Pacific trial 226 patients were randomized in a 2:1 ratio between sorafenib and placebo (5). Median overall survival was 6.5 months (95\% CI 5.56-7.56) in patients treated with sorafenib, compared with 4.2 months (3.75-5.46) in those who received placebo (hazard ratio [HR] 0.68 [95\% CI 0.50-0.93]; $\mathrm{p}=0.014)$. 
However, we are still far from an efficient control of HCC disease even if these results represent a landmark therapeutic advance in the treatment of a rather frequent tumour. The unsuccessful medical treatment of HCC is, at least in part, based on the complex molecular alterations present in HCC tissue and on the activation of multiple signal transduction pathways controlling cell proliferation and tumour progression (6). Angiogenesis is one of the prominent feature of $\mathrm{HCC}$ and is also one of the targets of sorafenib itself. In fact, the latter predominantly targets VEGF that plays a role from liver regeneration in cirrhosis to neo-vascularization in HCC typically expressing with portal and hepatic vein invasion in advanced phases. However, other growth factors and growth factor receptors like epidermal growth factor (EGF) and insulin-like growth factor (IGF) are involved in hepatocarcinogenesis (7). Based on these considerations, combination between different specific target-based agents is expected to improve the clinical benefits already obtained with sorafenib alone, but this is still a highly complex matter. In fact, despite some combinations being scientifically sound, toxicity remains as the main practical limitation, and safety data from phase I/II studies is mandatory prior to launching phase III initiatives (8). Therefore, the use of agents already known to be active in controlling the symptoms derived from HCC and/or cirrhosis and causing poor side effects could be useful in combinatory strategies. In this view, somatostatin and somatostatin analogs reduce the release of growth factors, such as IGF-1 or EGF, inhibit angiogenesis and through receptor interaction directly stop cell growth by inducing apoptosis or regulating cell cycle $(9,10)$. In vivo and in vitro expression of somatostatin receptors has been reported in HCC and especially the subtype 2 (11-13). Octreotide was previously used in HCC patients with conflicting results (14-21). We showed, in a previous study, that combination of octreotide and radiofrequency ablation 
produced about $80.0 \%$ of disease control and interesting mean overall survival (31.4 months) in a series of advanced HCC patients (22).

Based on these premises, a phase II multicenter study based on the combination between sorafenib and long acting octreotide (So.LAR) started in order to assess its safety and activity in advanced HCC.

\section{PATIENTS AND METHODS}

\section{Patient Selection}

Patients were required to have HCC confirmed by biopsy or diagnosed by radiological and clinical criteria. These criteria were hypervascular liver masses more than $2 \mathrm{~cm}$ and alfafetoprotein more than $400 \mathrm{ng} / \mathrm{dL}$. Other eligibility criteria were advanced disease not susceptible to local/surgical treatments, age at least 18 years, Child-Pugh $\mathrm{A}$ and compensated B, adequate hematologic (absolute neutrophil count $\geq 1.0 \times 10^{9} / \mathrm{L}$, platelet count of $\geq 60 \times 10^{9} / \mathrm{L}$ ), hepatic (total bilirubin $\leq 3 \mathrm{mg} / \mathrm{dL}$, albumin $\geq 2.5 \mathrm{mg} / \mathrm{dL}$ ) and renal function (serum creatinine $\leq 2 \mathrm{mg} / \mathrm{dL}$, urinary protein $<500 \mathrm{mg} / 24 \mathrm{~h}$ ). Patients were required to have at least one measurable lesion by CT-scan or MRI according to the RECIST criteria, performed within 4 weeks prior to start of therapy. HBV and HCV infection status at baseline was collected from medical history or laboratory tests.

Concomitant antiviral systemic therapy was allowed.

Exclusion criteria included an advanced second primary malignancy, significant medical comorbidities, clinically significant cardiovascular disease including 
uncontrolled hypertension, myocardial infarction and unstable angina, NYHA grade II or greater congestive heart failure, history of active bleeding.

The study protocol was approved by Local Ethical Committee and every patient gave written informed consent prior to study entry.

Patients underwent basal clinical evaluation, ECG, blood chemistry, analysis of liver function, $\alpha$-FP assay, whole body Computed Tomography, Esophagogastroduodenoscopy, echocardiography, and other examinations if clinically required.

\section{Treatment protocol}

Patients received sorafenib $400 \mathrm{mg}$ bid for 28 days with a following week of rest and long acting octreotide at a dose of $40 \mathrm{mg}$ every 28 days. Treatment was continued until disease progression or unacceptable toxicity. The first octreotide injection was administered ten days after sorafenib starting. Dose reduction of sorafenib (200 mg bid) and octreotide $(20 \mathrm{mg}$ ) were allowed for drug-related toxicities (National Cancer Institute Common Toxicity Criteria version 2.0). Dose delays or modifications were required for drug-related toxicities. For grade $3 / 4$ toxicities related to sorafenib, patients discontinued therapy until resolved, then restarted at $200 \mathrm{mg}$ bid with dose escalation if no toxicity occurred again. A modified scale was used for hand-foot syndrome (HFS) and specific dose modification were adopted (4). 


\section{Response assessment}

Response to treatment was assessed by at least two independent radiologists using RECIST criteria (23) every two months. At baseline, tumor lesions will be categorized measurable (lesions that can be accurately measured in at least one dimension [longest diameter to be recorded] as $\geq 20 \mathrm{~mm}$ with conventional techniques or as $\geq 10 \mathrm{~mm}$ with spiral CT scan) or non measurable (all other lesions, including small lesions [longest diameter $<20 \mathrm{~mm}$ with conventional techniques or $<10 \mathrm{~mm}$ with spiral CT scan] and truly non measurable lesions). All measurable lesions up to a maximum of 10 lesions in total should be identified as target lesions and recorded and measured at baseline. Taking into account the measurement of the longest diameter only for all target lesions, complete response was defined as the disappearance of all target lesions; partial response as at least a $30 \%$ decrease in the sum of the longest diameter of target lesions, taking as reference the baseline sum longest diameter; progressive disease as at least a $20 \%$ increase in the sum of the longest diameter of target lesions, taking as reference the smallest sum longest diameter recorded since the treatment started or the appearance of one or more new lesions; stable disease when neither sufficient shrinkage to qualify for partial response nor sufficient increase to qualify for progressive disease, taking as reference the smallest sum longest diameter since the treatment started. The diseasecontrol rate was defined as the percentage of patients who had a best-response rating of complete response, partial response, or stable disease (according to RECIST) that was maintained for at least 28 days after the first demonstration of that rating on the basis of independent radiologic review. 


\section{Study Design and Statistical Analysis}

Simon's (24) optimum two-stage Phase II design was used in this trial. Assuming the target and lower activity of the combination to be $20 \%$ and $10 \%$, respectively, 21 patients are required in the first stage of accrual and, if there are more than one objective responses in the first stage, a total of 50 patients will be accrued.

The time to progression (TTP) was calculated as the period from the date of starting treatment to the first observation of disease progression within 60 days after the start of treatment or the most recent tumor assessment. The overall survival (OS) time was calculated as the period from the date of starting treatment until death from any cause or until the date of the last follow-up, at which point data were censored. TTP and OS were both determined by Kaplan-Meier product-limit method (25). SPSS software (version 13.05, SPSS, Chicago) was used for statistical analysis. A P value of less than 0.05 was considered to indicate statistical significance.

\section{RESULTS}

\section{Patient Characteristics}

Fifty patients were enrolled between July 2007 and July 2008. Patients' characteristics are summarized in table 1. Eighty-six percent of patients were male with a median age of 67.5. Chronic hepatitis $\mathrm{C}$ virus infection was the predominant cause of liver disease, $34(68 \%)$ patients, followed by chronic hepatitis B virus infection and alcohol consumption. The majority of patients (39 out 50) were Child A. Twenty-nine patients were classified as C by BCLC staging classification. 
Sixteen patients out of $50(34 \%)$ were naïve from other therapies, while all the others were previously treated with local and/or systemic treatments. Specifically, 10 out of 50 patients $(20 \%)$ were pre-treated with systemic therapies.

Median treatment duration was 5 months (range 1-14 months). Sixteen out of 50 patients $(34 \%)$ received $\leq 3$ months of treatment.

\section{Clinical activity}

We recorded 5 PRs (10\%), 33 SDs (66\%) and 12 PDs (24\%). Overall disease control rate $(\mathrm{CR}+\mathrm{PR}+\mathrm{SD})$ was $76 \%$ (Table 2). Fifty-two per cent $(26 / 50)$ of patients had a stable disease for 4 months or longer. A panel of independent radiologists examined the radiograms and, most frequently, lesions appeared unchanged, but tumour necrosis increased (Figure 1). Smaller lesions appeared to change in response to treatment easier than larger tumour lesions. A patient, who achieved PR, maintained the response up to twelve months (Figure 1); among the 36 patients that obtained a stable disease, fourteen of them maintained this result for up to six months. Interestingly, two out of five patients with a PR and 27 (75\%) out of 36 patients who had a SD had a HCV chronic infection (Tables 1 and 2).

Response and survival did not seem to be directly correlated. In fact, three patients with SD and 1 with PR died after cirrhosis progression (encephalopathy, variceal bleeding, ascitis).

Median time to progression (TTP) was 7.0 months (95\% CI, 3.0 to 10.9 months) and median overall survival was 12.0 months (95\% CI, 6.3 to 17.4 months) (fig.2 A and $\mathrm{B}$, respectively). 
Previous treatments, basal alpha fetoprotein levels, one or multiple liver lesions were not correlated to both OS and TTP (Fig. 3 and 4).

\section{Toxicity}

Acute side-effects are summarized in Table 3. Treatment was generally well tolerated. Most common side effects were diarrhoea (32\%) and hand-foot syndrome (10\%). Grade III diarrhoea developed in 3 patients, hypertension and hand-foot syndrome in two patients, and proctalgia in one patients. This effect was not strictly related to therapy, but to a pre-existing condition of portal hypertension in cirrhotic patients. Most common grade II toxicity was diarrhoea in about 9.3\%. Less frequent effects were hypertension, bleeding, proctalgia and abdominal pain (Table 3).

Sorafenib dose reduction was required temporarily in 15 out of 50 patients. Octreotide dose reduction (50\% dose reduction) was required in all patients reporting grade 3 diarrhoea and grade 2 recurrent diarrhoea. In details, for any grade 1 toxicities no dose modification was adopted. For grade 2 diarrhoea, sorafenib dose was modified only if supportive drugs (i.e. loperamide, racecadotril, and so on) were unable to control diarrhoea. For grade 3 diarrhoea sorafenib was stopped until the side effect resolved, then was restarted at $400 \mathrm{mg}$ for one week and at full doses if no problems. For hand foot syndrome dose delays and modifications were adopted according to Abou-Alfa et al. (26). Fatigue was managed with supportive drugs. For grade 3 hypertension cardiovascular drugs were used as needed. Discontinuation of sorafenib occurred in $13.9 \%$ of patients because of adverse events. However, all patients recovered full doses after toxicity disappeared. Moreover, no toxicity-related death was recorded. 


\section{DISCUSSION}

HCC prognosis is very poor without specific treatments, and the median survivals for patients with early and advanced tumors are 6-9 months and 1-2 months, respectively. Reported median survival for treated patients with stage B-C by Barcelona Clinic Liver Cancer (BCLC) classification is 11-20 months. Apart from tumour progression, functional liver impairment due to cirrhosis significantly influences the outcome of the patients. Moreover, the treatment of liver cancer in an already-damaged normal cirrhotic tissue increases pharmacological challenges. The significant risk of toxicity by systemic chemotherapy is not counter-acted by significant response rate; in fact, single agents like doxorubicin and mytomicin $\mathrm{C}$ do not achieve a response rate higher than $20 \%$ and active multidrug-regimens may be heavily toxic with reported treatment-related deaths (27).

The concept of targeted-therapies emerged to be a promising approach for the innovative and effective medical treatment of different cancers, including HCC $(1,2)$. In this scenario, the landmark SHARP study established sorafenib as new standard of treatment for HCC (4). However, HCC remains a challenging disease and new agents/regimens have to be assessed to improve the results obtained with sorafenib alone. On the other hand, octreotide has been already used with contrasting results in the treatment of HCC. The present study is an attempt to disclose if octreotide and sorafenib may represent a valuable therapeutic option for advanced HCC patients. The schedule of sorafenib used in the present study was similar to that one of other studies 
and was chosen to allow a better patient compliance considering also the performance of the patients enclosed in the study (28-30). Forty mg octreotide was chosen because we have preliminary observations that suggest that it is well tolerated by the patients affected by advanced HCC (unpublished results).

Bevacizumab-based regimens in HCC produce a median PFS/TTP ranging from 4.1 to 9 months and phase II studies with Epidermal Growth Factor receptor inhibitors reported a median PFS/TTP ranging from 1.36 to 4.5 months (31). Median OS is more than 10 months with regimens based on erlotinib (32,33) and sorafenib (4) administered as single agents. The $10 \%$ overall response rate and the $74 \%$ disease control rate reported in the present study appear encouraging. The median PFS of 7.0 months and the mean OS of about 12 months reported in the present study with sorafenib and octreotide combination is favourably comparable with the previous cited target-based treatments. These results appear better than others obtained in previous trials; the possible explanation is likely due also to the punctual surveillance for adverse events that guaranteed an adequate dose density, that is the institution of an out-patients clinic to monitor any toxicities possibly related to treatment with weekly appointments.

Moreover, in the present study $22 \%$ of patients were classified as B by Child and, differently from most of the studies evaluating novel targeted drugs, $23 \%$ of patients were previously treated by medical therapies. In the present study, portal vein thrombosis was the only factor significantly related to poor OS, while neither multiple liver lesions nor previous treatments had statistically significant impact on survival. Interestingly, portal vein thrombosis occurrence was not correlated to TTP and this finding is in agreement with the well known ability of this prognostic factor to predict the survival but not the response to therapy (34). Yau et al. recently treated with 
sorafenib 51 patients (35). In that study, $4(8 \%)$ patients achieved partial responses, and $9(18 \%)$ patients had stable disease for at least 12 weeks. The median OS was 5 months and patients without extrahepatic spread, particularly without lung metastasis $(\mathrm{P}<.01)$, were more likely to benefit from sorafenib treatment. Interestingly, in our series about $30 \%$ of patients had extra-hepatic disease spreading.

Treatment was generally well tolerated as we didn't observed any grade IV toxicity or life-threatening gastro-intestinal haemorrhage. The most common side-effects, i.e. diarrhoea, may be related to both drugs. However, the rate of less than $7 \%$ of grade III diarrhoea was comparable and lower to that reported by octreotide (19) and sorafenib alone, respectively (4). Recently, another trial based on the combination between erlotinib and bevacizumab has been reported with 10 out of 40 PRs, a median OS of 15.65 months and a median TTP of 9.0 months. In our study, the number of PRs was limited and the survival of the patients lower. However, it should be underlined that only $12.5 \%$ of patients were Child B and 1 patient was ECOG PS 2 in the study by Thomas et al. On the other hand, Thomas et al. reported several grade IV toxic effects and relevant grade III toxicities including hypertension, diarrhoea and fatigue. The low toxicity observed in our series could be explained on the basis of the following considerations: i) strict surveillance for side effects; ii) possible palliation by octreotide of the metabolic effects induced by hepatopathy; iii) one week withdrawal of sorafenib administration.

In conclusion, sorafenib plus octreotide is a safe and effective option in advanced HCC patients with compromised metabolic scores and/or low PS. The exact contribution of each drug is difficult to define and may be the aim of future randomized studies. The chance to identify and select patients according to clinical and molecular predictors of 
sorafenib- and octreotide-mediated clinical benefits and mechanisms of resistance may probably represent the direction to improve the obtained results.

\section{REFERENCES}

1. Addeo R, Caraglia M, Del Prete S. (2009). Highlights of regional meeting of Italian Southern Oncological Group (GOIM): focus on hepatocellular carcinoma: biological and clinical background, therapeutic guide-lines and perspectives. 7 November 2008, Naples, Italy. Expert Opin Investig Drugs.;18(3):373-378.

2. Caraglia M, Tassone P, Marra M, et al (2006). Targeting Raf-kinase: molecular rationales and translational issues. Ann Oncol.; 17 Suppl 7:vii124-127

3. Takimoto CH, Awada A. (2008). Safety and anti-tumor activity of sorafenib (Nexavar®) in combination with other anti-cancer agents: a review of clinical trials. Cancer Chemother Pharmacol; 61:535-548

4. Llovet JM, Ricci S, Mazzaferro V et al. (2008). SHARP Investigators Study Group Sorafenib in advanced hepatocellular carcinoma N Engl J Med. 24;359:378-90.

5. Cheng AL, Kang YK, Chen Z, et al. (2009). Efficacy and safety of sorafenib in patients in the Asia-Pacific region with advanced hepatocellular carcinoma: a phase III randomised, double-blind, placebo-controlled trial. Lancet Oncol.;10:25-34.

6. Thomas MB, Abbruzzese JL. (2008). Opportunities for targeted therapies in hepatocellular carcinoma. J Clin Oncol; 23:8093-8108

7. Llovet JM, Bruix J. (2008) Molecular targeted therapies in hepatocellular carcinoma. Hepatology; 48:1312-1327. 
8. Llovet JM, Di Bisceglie A, Bruix J, et al. (2008). Design and end-points of clinical trials in HCC. J Natl Cancer Inst;100:698-711.

9. Buscail L, Vernejoul F, Faure P, Torrisani J, Susini C. (2002) Regulation of cell proliferation by somatostatin Ann Endocrinol (Paris),;63:2S13-8;

10. Hagemeister AL, Sheridan MA (2008) Somatostatin inhibits hepatic growth hormone receptor and insulin-like growth factor I mRNA expression by activating the ERK and PI3K signaling pathways Am $\mathrm{J}$ Physiol Regul Integr Comp Physiol.;295:R490-7.

11. Zhao M, Laissue JA, Zimmermann A. (1993). "Neuroendocrine" differentiation in hepatocellular carcinomas (HCCs): immunohistochemical reactivity is related to distinct tumor cell types, but not to tumor grade. Histol Histopathol ; 8 :617-26.

12. Kouroumalis E, Skordilis P, Thermos K, et al. (1998). Treatment of hepatocellular carcinoma with octreotide: a randomized controlled study. Gut; 42:442-7.

13. Reubi JC, Zimmermann A, Jonas S et al. (1999). Regulatory peptide receptors in human hepatocellular carcinomas. Gut; 45 :766-74.

14. Siveke JT, Herberhold C, Folwaczny C. (2003). Complete regression of advanced HCC with long acting octreotide. Gut; 52:1531.

15. Kouroumalis E, Skordilis P, Thermos K et al. (1998). Treatment of hepatocellular carcinoma with octreotide: a randomized controlled study. Gut; 42:442-7.

16. Samonakis DN, Moschandreas J, Arnaoutis T et al. (2002). Treatment of hepatocellular carcinoma with long acting somatostatin analogues. Oncol Rep; 9:903907. 
17. Dimitroulopoulos D, Xinopoulos D, Tsamakidis K et al. (2002)The role of Sandostatin LAR in treating patients with advanced hepatocellular cancer. Hepatogastroenterology; 49:1245-1250.

18. Raderer M, Hejna MH, Muller C et al. (2000) Treatment of hepatocellular cancer with the long acting somatostatin analog lanreotide in vitro and in vivo. Int $\mathrm{J}$ Oncol, 16:1197-1201.

19. Cebon $\mathbf{J}$ and Australasian Gastro-Intestinal Trials Group (AGITG) AG000IH Investigators (2006). Somatostatin receptor expression, tumor response, and quality of life in patients with advanced hepatocellular carcinoma treated with long-acting octreotide. Br J Cancer; 95:853-861.

20. Becker G, Allgaier HP, Olschewski M et al. (2007). HECTOR Study Group. Longacting octreotide versus placebo for treatment of advanced HCC: a randomized controlled double-blind study. Hepatology; 45: 9-15.

21. Samonakis DN, Notas G, Christodoulakis N, Kouroumalis EA. (2008). Mechanisms of action and resistance of somatostatin analogues for the treatment of hepatocellular carcinoma: a message not well taken. Dig Dis Sci.; 53: 2359-65.

22. Montella L, Addeo R, Caraglia M, et al. (2008) Vascular endothelial growth factor monitoring in advanced hepatocellular carcinoma patients treated with radiofrequency ablation plus octreotide: A single center experience. Oncol Rep.;20:385-90.

23. Therasse P, Susan G. Arbuck SG et al. (2000) New Guidelines to Evaluate the Response to Treatment in Solid Tumors. J Natl Cancer Inst; 92:205-16.

24. Simon R (1989). Optimal two-stage designs for phase II clinical trials. Control Clin Trials ; 10: 1-10. 
25. Kaplan E and Meier P. (1958) Nonparametric estimation from incomplete observations. J Am Stat Assoc; 53:457-481.

26. Leung TWT, Patt YZ, Lau W et al. (1999) Complete pathological remission is possible with systemic combination chemotherapy for inoperable hepatocellular carcinoma. Clin Cancer Res; 5:1676-81.

27.Abou-Alfa GK, Schwartz L, Ricci S, Amadori D, Santoro A, Figer A, De Greve J, Douillard J-Y, Lathia C, Schwartz B, Taylor I, Moscovici M, Saltz LB. Phase II study of sorafenib in patients with advanced hepatocellular carcinoma. J Clin Oncol 2006; 24: 4293-4300

28. Minami H, Kawada K, Ebi H, Kitagawa K, Kim YI, Araki K, Mukai H, Tahara M, Nakajima H, Nakajima K. (2008) Phase I and pharmacokinetic study of sorafenib, an oral multikinase inhibitor, in Japanese patients with advanced refractory solid tumors. Cancer Sci.;99:1492-8;

29.Furuse J, Ishii H, Nakachi K, Suzuki E, Shimizu S, Nakajima K. (2008) Phase I study of sorafenib in Japanese patients with hepatocellular carcinoma. Cancer Sci.;99:159-65;

30.Strumberg D, Clark JW, Awada A, Moore MJ, Richly H, Hendlisz A, Hirte HW, Eder JP, Lenz HJ, Schwartz B. (2007) Safety, pharmacokinetics, and preliminary antitumor activity of sorafenib: a review of four phase I trials in patients with advanced refractory solid tumors. Oncologist.;12:426-37

31. Faivre SJ, RE, Douillard J, et al (2007) Assessment of safety and drug-induced tumor necrosis with sunitinib in patients (pts) with unresectable hepatocellular carcinoma (HCC). J Clin Oncol 25:149s, (suppl; abstr 3546). 
32. Zhu AX. (2008). Development of sorafenib and other molecularly targeted agents in hepatocellular carcinoma. Cancer; 112:250-9

33 Philip PA, Mahoney MR, Allmer C, et al. (2005) Phase II study of erlotinib (OSI774) in patients with advanced hepatocellular cancer. J Clin Oncol;23:6657-6663.

34. Thomas MB, Chadha R, Glover K et al. (2007). Phase 2 study of erlotinib in patients with unresectable hepatocellular carcinoma. Cancer; 110:1059-66

35. Yau T, Chan P, Ng KK, et al. (2009). Phase 2 open-label study of single-agent sorafenib in treating advanced hepatocellular carcinoma in a hepatitis B-endemic Asian population: presence of lung metastasis predicts poor response. Cancer;115:428-36 
Table 1. Characteristics of the enrolled patients

No of patients

Sex (Male/Female \%)

Range Age, years,

Median age

Positive Hepatitis status (no/\%)

-Hepatitis B

-Hepatitis C

-Hepatitis B and C

PS (ECOG)

0

1

2

No of liver lesions (no/\%)

$-1$

$-2$

-multiple

-extrahepatic disease without liver lesions

-thrombosis

Metastatic disease

-Lung

-Lymph nodes

-Bone

-Adrenal Gland

-Resected medullary lesion

Child-Pugh A/B

BCLC B/C

Basal a-FP>VN (no/\%)

Esophageal varices (no/\%)

Prior treatments

-surgical resection

-liver transplantation

-chemoembolization

-radiofrequency ablation and/or percutaneous ethanol injection

-systemic therapies
50

$43 \mathrm{M} / 7 \mathrm{~F}, 86 \% / 14 \%$

$50-82$ years

67.5

$11(22 \%)$

$34(68 \%)$

$1(2 \%)$

26

20

4

$9(18 \%)$

$8(18 \%)$

$31(62 \%)$

$2(4 \%)$

$14(28 \%)$

$16(32 \%)$

$4(8 \%)$

$5(10 \%)$

$4(8 \%)$

$2(4 \%)$

$1(2 \%)$

$39 / 11$

$21 / 29$

$31(62 \%)$

$26(52 \%)$

(no/\%)

$11(22 \%)$

$1(2 \%)$

$15(30 \%)$

$15(30 \%)$

$11(22 \%)$ 
Table 2. Evaluation of activity of Sorafenib plus Octreotide combination treatment at 14 months

\begin{tabular}{|l|c|c|}
\hline & No. of patients & $\%$ \\
\hline Complete Response & - & \\
\hline Partial Response & 5 & 4 \\
\hline Stable Disease & 33 & 72 \\
\hline Overall Disease control & 38 & 76 \\
\hline Progression Disease & 12 & 28 \\
\hline
\end{tabular}


Table 3. Number of Adverse Events Recorded Among the Entire Study Classified for Toxicity Grade Accordingly to the NCI-CTC and Number of Patients in Which Each Adverse Event Was Recorded

\begin{tabular}{|c|c|c|c|c|c|}
\hline & $\begin{array}{c}\text { Grade } 1 \\
(\%)\end{array}$ & Grade 2 & Grade 3 & Grade 4 & $\begin{array}{l}\text { No of } \\
\text { patients } \\
(\%)\end{array}$ \\
\hline \multicolumn{6}{|l|}{$\begin{array}{l}\text { Gastrointestinal } \\
\text { symptoms }\end{array}$} \\
\hline -diarrhoea & $6 \%$ & $20 \%$ & $6 \%$ & & $16(32 \%)$ \\
\hline \multicolumn{6}{|l|}{$\begin{array}{l}\text { Dermatological } \\
\text { symptoms }\end{array}$} \\
\hline - dry skin & $2 \%$ & & & & $1(2 \%)$ \\
\hline - hand-foot syndrome & $4 \%$ & $2 \%$ & $4 \%$ & & $5(10 \%)$ \\
\hline - alopecia & $2 \%$ & & & & $1(2 \%)$ \\
\hline \multicolumn{6}{|l|}{$\begin{array}{l}\text { Constitutional } \\
\text { symptoms }\end{array}$} \\
\hline -fatigue & $2 \%$ & & & & $1(2 \%)$ \\
\hline -hypertension & $2 \%$ & $4 \%$ & $4 \%$ & & $5(10 \%)$ \\
\hline $\begin{array}{l}\text {-voice } \\
\text { changes/hoarseness }\end{array}$ & $4 \%$ & & & & $2(4 \%)$ \\
\hline -abdominal pain & $2 \%$ & $2 \%$ & & & $2(4 \%)$ \\
\hline -bleeding & $6 \%$ & & & & $3(6 \%)$ \\
\hline -proctalgia & $2 \%$ & & $2 \%$ & & $2(4 \%)$ \\
\hline -arthralgia & $2 \%$ & & & & $1(2 \%)$ \\
\hline -hypothyroidism & $2 \%$ & $2 \%$ & & & $2(4 \%)$ \\
\hline
\end{tabular}




\section{Figure legends}

\section{Figure 1.}

Kaplan-Meier estimate of (A) time to progression and (B) overall survival of the patients from study entry.

\section{Figure 2.}

Kaplan-Meier estimate of the overall survival time of the patients stratified for (A) previous treatment or (B) serum alpha-fetoprotein levels or (C) multiple organs involvement or (D) portal vein thrombosis. P-values were derived from a log rank test.

\section{Figure 3.}

Kaplan-Meier estimate of the time to progression of the patients stratified for (A) previous treatments or (B) serum alpha-fetoprotein levels or (C) multiple organs involvement or (D) portal vein thrombosis. P-values were derived from a log rank test. 